\title{
Design and Evaluation of Simple UI for Mobile Healthcare Application
}

\author{
Atsushi Ito
}

\begin{abstract}
In 2008, the Japanese government enacted a new healthcare law that requires companies to reduce the weight of overweight employees suffering from so-called metabolic syndrome, and the weight of their spouses. In 2013, companies that fail to reduce the weight of their employees and their spouses will be penalized. So, companies aim to introduce tools to support to loose weight. At first, we developed the first version of mobile application, named HIPPO (Healthcare Information Platform Project), to support to loose weight in 2007. This application worked well during two months and $76 \%$ of users whose BMI was 25 or over lost their weight. However, this problem was that many users could not continue to use this application and users who could continued to use this application could loose weight. We thought that to develop application that made user continue to use was important. So, in 2008 , redesigned the system architecture and application from three view points, (1) Address real user needs, (2) Rethink and streamline the data-entry model for food and exercise, (3) Make the user experience personal and engaging. We designed one simple application with limited functions and use email as a support tool. As the result, $84 \%$ of uses could continue to use the second generation application.
\end{abstract}

Keywords-mobile healthcare application, weight control, metabolic syndrome, mobile persuasion

\section{INTRODUCTION}

$\mathbf{I}$ N 2008, the Japanese government enacted a new healthcare law that requires companies to reduce the weight of overweight employees, suffering from so- called metabolic syndrome, and the weight of their spouses. So, companies aim to introduce tools to support dieting. This regulation uses the Body Mass Index (BMI) to evaluate healthy body weight as described in Tab. I. It is important to reduce the number of employees and their spouses whose BMI is over 25 by $10 \%$ [1]. However, employees are very busy and lack time to take care of their health. For this reason, an effective healthcare method is required, especially to help tackle metabolic syndrome. As mobile phones are ubiquitous and very useful in supporting behavior modification in our daily lives, we developed a mobile healthcare application. It is not easy to realize effective healthcare applications for mobile phones, and there is no application in wide use in society. In this paper, we explain our healthcare application and the results of our evaluation. First, we explain our first generation mobile healthcare application and describe the problems that we found based on the results in Section 2. Then we explain the process of reforming the mobile healthcare application and the results of the trial in Section 3. In Section 4, we conclude this paper and discuss further improvements to the mobile healthcare application.

A. Ito is with the KDDI Research and Development Laboratories, Tokyo, 102-8460, Japan (e-mails: at-itou@kddi.com or at.ito@mac.com).
TABLE I

DEFINITION OF BMI

\begin{tabular}{|c|c|}
\hline BMI (Body Mass Index) & Condition \\
\hline \hline$<18.5$ & Lean \\
\hline $18.5 \leq$ and $<25$ & Standard \\
\hline$\leq 25$ & Fat \\
\hline
\end{tabular}

\section{RELATED WORKS}

There are several activities and reports relating to mobile healthcare supported by ICT. However, it is very difficult to be widely used and they are not so popular yet. Some ICT giants have services [2], [3], but some are already closed [2]. These services are focusing on to keep user healthy and to provide variety of functions, however not directly discussing on how to keep users to use these services. We would like to focus on how to increase continuity of mobile healthcare application. Some manufacturers of health care equipment have their own services [4], [5]. There is an activity to standardize communication between bio medical equipment and PC/cloud [6]. These services are focusing on selling their devices, so it does not meet the heterogeneous environment. The devices that can be connected ICT devices were not so many and it is not easy to connect every device even if [6] are realized. So that, we dis not considered to connect devices. Also there is an open source project, named Paco, to develop cloud services for healthcare easily [7]. The Paco is very simple and easy to use, however, it is web based system and not suitable to develop attractive user interface. We decided to develop more colorful and graphical application. In addition, there are new ideas on explaining and designing effective implementation of healthcare applications especially for changing behavior [8]. Based on this idea, some healthcare applications have been implemented [9], [10]. Also there is a unique experiment to use texting on mobile phone for healthcare [11]. Some of them are developed as a kind of caring games, but it might not be suitable variety of users. Also, texting might be too simple to attract people. Therefore, at the development of the first generation applications, we decided to develop limited functions and asked users to select to use preferable one. At last, we developed simpler application as the second generation application that had a few mandatory functions to keep motivation of users.

\section{Development and Evaluation of the First Generation Mobile HealthCARE Application}

In this section, we outline our first generation mobile healthcare applications. To realize the mobile healthcare system, we 


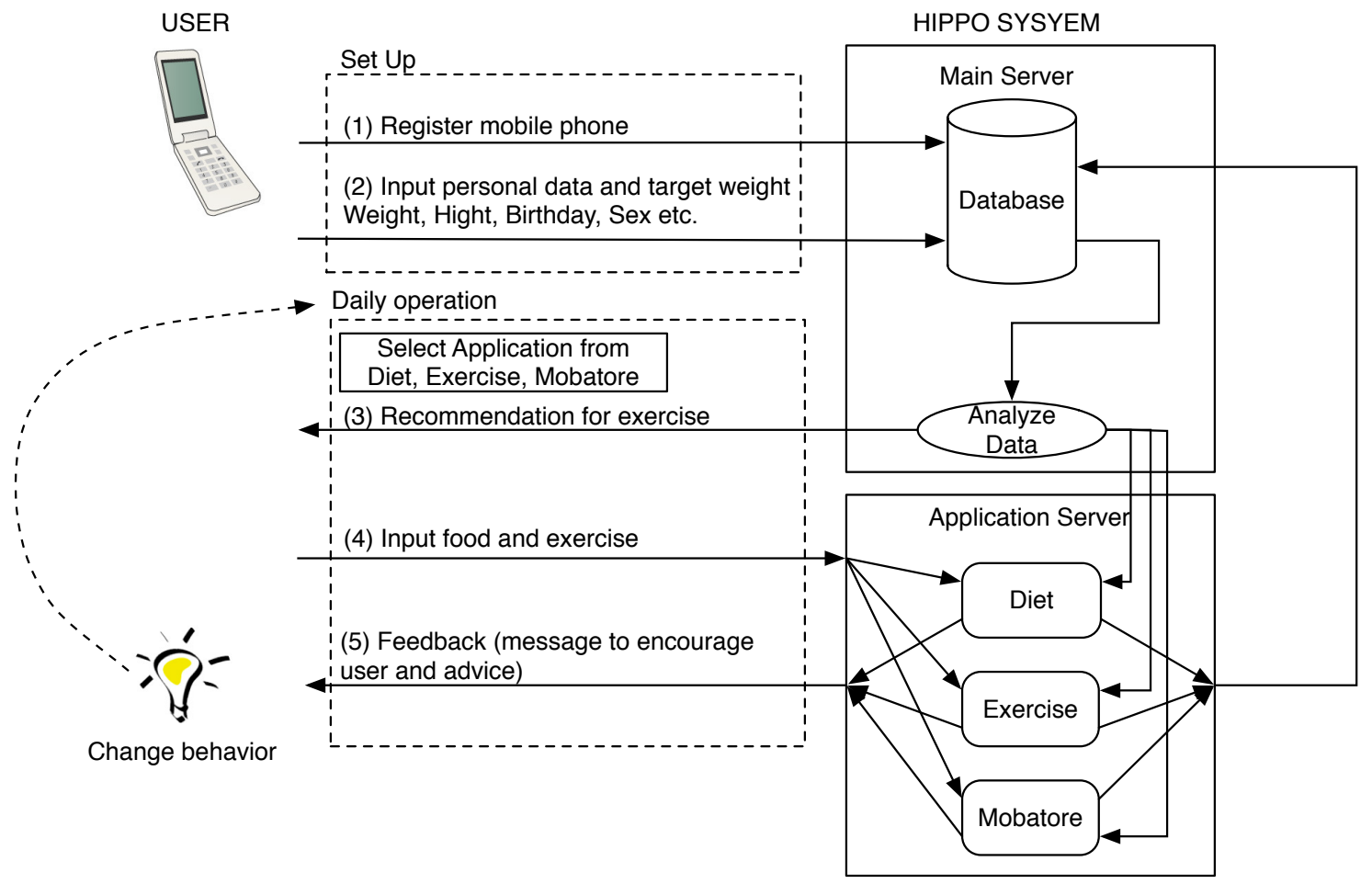

Fig. 1. System design of the first generation mobile health care system

designed the Healthcare Information Platform Project (HIPPO) to cover three areas: Food, Exercise and Support Network. First we developed three applications to support companies in helping their employees stay slim and healthy:

1) HIPPO Diet: plan, input, track and view food intake patterns (nutrition, variety, calories)

2) HIPPO Exercise: plan, input, track and view exercise activities

3) HIPPO Mobatore (Mobile training): social network service to accompany Diet and Exercise application

\section{A. Designing the First Generation Mobile Healthcare System and Applications}

Figure 1 shows an outline of our mobile healthcare system. This system consists of a database to accumulate data, analyze data function, and three applications. The database consists of personal data, birthday, hight, weight, sex etc. and daily activity, foods and exercise. These data is used to calculate BMI and set up weight reduction plan.

Firstly, (1) resister a mobile phone on the databank. Then, (2) input personal data such as height, weight and birthday to the databank. Then set the target weight that will be used in each application.

After this step, set up is finished and a user is into the daily use mode. For the daily use, each user should select an application first for the users purpose and intention. or example, a user may choose "Diet" at the lunch, "Exercise" after walking and "Mobatore" to share workout information with friends. (3) Every day, a recommendation of exercise is provided. This recommendation is calculated in the main server from the balance of food and exercise information. (4) Every day, users are required to input what they eat and how much they do exercise. (5) The application platform provides feedback. It displays the recommendation and current status of BMI, and also sends emails to users giving encouragement, requests to input information and information about a prize for the person who exercises the most, etc.

For inputting the record of exercise, we provided the Exercise application. Users can input details of the exercise, such as 20 minutes jogging etc. This application selects a rival automatically and informs the user how much weight their rival lost. To input food, we provided the Diet application. This application displays recommended food, which includes what the user should eat and the vitamin and mineral balance on a radar chart. For communicating with friends, we provided the SNS function, Mobatore (mobile training). This application provides a function for inputting the users exercise history, making a blog and sending mail to friends. We intended to support communication among users for mutual encouragement.

These applications were designed for a mobile phone (not a smart phone). Diet and Exercise were implemented as Web services and Mobatore was implemented as a Flash application (we used Flash 2.0) to be used on many mobile phones. Details of the implementation is described in Tab. II.

\section{B. Trial and Results}

1) Outline of trial: An in-house trial of the mobile healthcare applications was held from February 12, 2008 to March 31. We announced the trial to all 11,764 KDDI 
TABLE II

IMPLEMENTATION

\begin{tabular}{|c|c|c|c|}
\hline Handset & $\begin{array}{l}\text { SW } \\
\text { HW }\end{array}$ & $\begin{array}{l}\text { Application } \\
\text { Mobile Phone }\end{array}$ & $\begin{array}{c}\text { BREW } 3.1 \text { or higher [12] } \\
\text { Flash Lite } 2.0 \text { [13] } \\
\text { Three Japanese carriers }\end{array}$ \\
\hline Server & HW & $\begin{array}{c}\text { OS } \\
\text { Database } \\
\text { Web Server } \\
\text { CGI } \\
\text { CPU } \\
\text { RAM }\end{array}$ & $\begin{array}{c}\text { RedHat Enterprise Linux ES } 4 \\
\text { PostgresSQL } 8.0 \\
\text { Apache } 2.0 \\
\text { Perl } 15.8 \\
\text { Intel Core2Duo } 2.66 \mathrm{GHz} \\
4 \mathrm{~GB}\end{array}$ \\
\hline
\end{tabular}

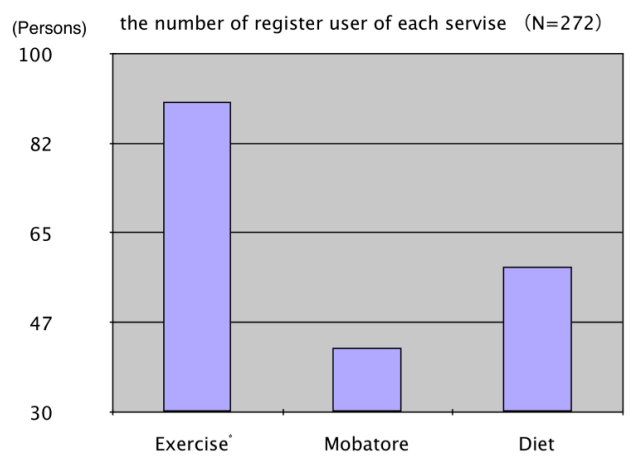

Fig. 2. The number of users of applications.

employees and requested that they register on our mobile healthcare system. Out of the 308 employees we recruited, 278 employees (2.4\% of total employees) remained at the end of March (227 male and 51 female). At the end of the trial, we sent questionnaires to the trial users.

2) The number of users of each application: Figure 2 displays the number of users of each application. Exercise is the most popular. We supposed that this application was appealing because it is simpler to use than the other applications.

3) Changing weight: Of the 278 registered users, 114 lost weight. The number of users whose BMI was 25 or over was 75. Weight change was observed in 33 users. In the 33 users, $76 \%$ lost weight (Fig. 3). We also checked the percentage of weight loss by group of BMI over 23 and BMI over 22. The results were similar.

4) Continuity: The trial period was the 48 days from February 12 to March 31, 2008. Average continuity days were 19.8. At the beginning of trial, many users dropped out. The reason for the short continuity was the complexity of the operation (Fig. 4). Figure 5 displays the average continuity days of each application. Exercise and Diet were supported, but Mobatore was not supported. We suppose that SNS of healthcare is not supported since it includes private information.

5) Answers to questionnaire: After the trial, we asked trial users to answer the questionnaire, and 87 users answered. (1) What was your reason for joining the trial? Take care of health (Keep Health Condition and Reduce Weight) was 67\%, so the majority was interested in their health (Tab. III).

(2) When and where did you use this application? Free time and On the train were the most popular answers (Fig. 6). On

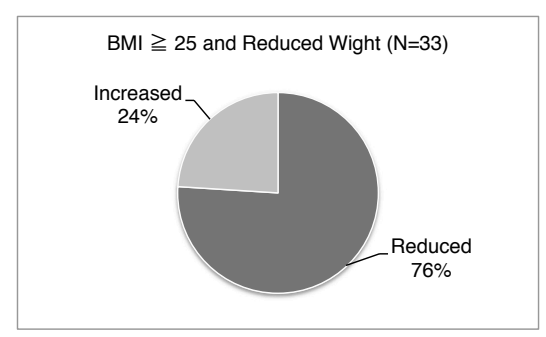

Fig. 3. Percentage of reduced weight.

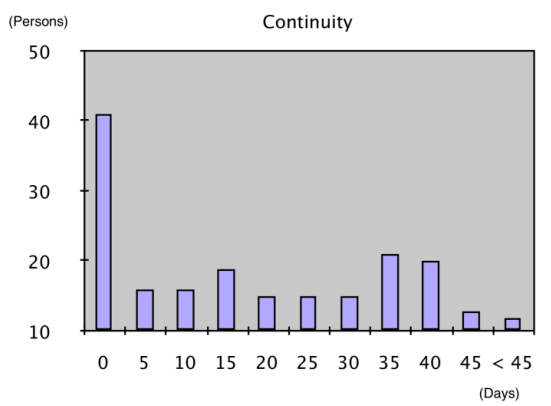

Fig. 4. Continuity of use.

TABLE III

REASON TO JOIN THE TRIAL

\begin{tabular}{|c|c|}
\hline Reason & $\boldsymbol{\%}$ \\
\hline \hline Keep Health Condition & 39 \\
\hline Reduce Weight & 28 \\
\hline Interested In & 25 \\
\hline Recommended from Friends & 2 \\
\hline Other & 6 \\
\hline
\end{tabular}

the train might be a specific answer to Tokyo. The application should be designed considering life style.

(3) Prompted to consider health? $65 \%$ of users thought that they were prompted to consider their health. By using the mobile health care application, they were prompted to take care of their health (Fig. 7)

\section{Evaluation of the First Generation Application}

As a result, about $20 \%$ of trial users input data every day and $25 \%$, of trial users input data once a week or more. Forty percent of trial users used this application in their free

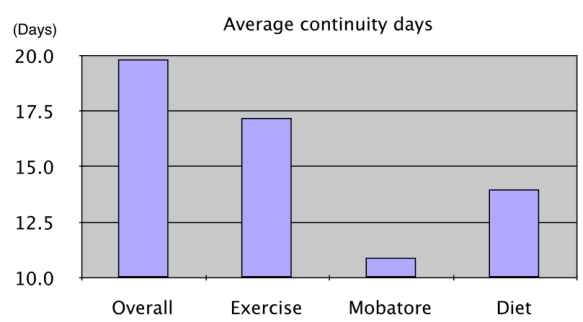

Fig. 5. Average continuity days of each application. 
When did you used HIPPO? (multiple answers are allowed $\mathrm{N}=87$ )

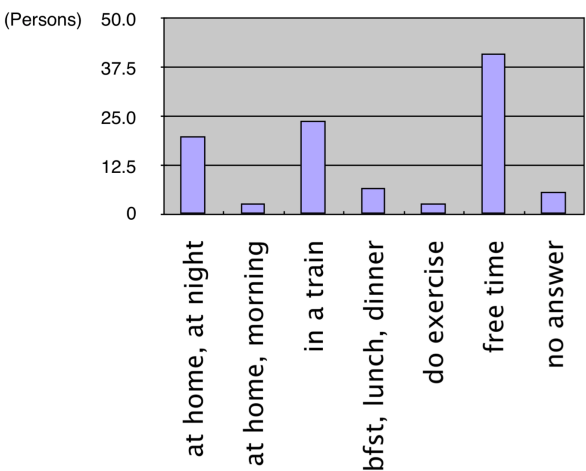

Fig. 6. When and where did you used applications.

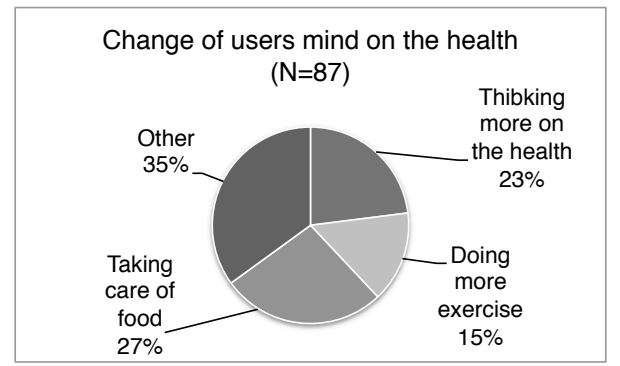

Fig. 7. Change of users mind on health.

time at home or in the office and $25 \%$ of users used the application on the train. In Japan, commuters spend about one hour a day traveling between homes and offices by train. Sixtyfive percent of users modied their behavior to improve their health condition by using this application. Seventy-five users had a BMI over 25. In that group, 30\% (25 users) lost weight. Based on these results, we concluded that a mobile phone is useful for healthcare. In particular, two-way communication might be useful for realizing changes in behavior. However, few people used this application as described in Fig. 5. We analyzed the results of the trial and concluded that we need to reduce the dropout rate and provide greater motivation to users to establish more permanent behavior habits. As we open our mobile phone and check email, the healthcare application should provide easy and simple operation. That is what we learn from the first trial.

\section{Second Generation Mobile Healthcare APPLICATIONS}

Based on analysis of the user experience of the first generation HIPPO applications, information from the HIPPO inhouse trial as well as from interviews with HIPPO users, we formed a set of insights regarding the needs and opportunities for HIPPO. These insights have formed the basis of the redesign.

1. Address real user needs

Besides addressing companies needs, focus the design of the HIPPO application around persons and key scenarios
- Users who want to lose weight have different goals and needs than those who want to exercise more.

- Design with scenarios in mind, to optimize the experience for key activities (e.g. enter information, view progress)

2. Rethink and streamline the data-entry model for food and exercise

- Consider fast and efficient input methods, allow data entry in stages (rough first, add details later); automatic data input; optimize options and menus.

3. Make the user experience personal and engaging

- An inviting, cohesive look and feel

- Information and recommendations feel personal and relevant

- Engage on a deeper level - people gain value from using the application

The following three sections explain the reforming of our mobile healthcare application.

\section{A. The Data Input Model}

Improving the data input experience is not about adding more. Careful design of the task flow, layout and providing meaningful visuals will make people feel confident, comfortable and enable them to use the application efficiently.

\section{B. A Personal and Engaging UI}

People's time is precious. They need to choose carefully where to invest their time and energy. Especially for topics such as health, weight and wellbeing, a personal and engaging approach is expected. The new HIPPO experience:

- is quick. The UI is simple, visual and direct.

- is desirable and dynamic. It is engaging.

- is meaningful and informative. Messages and recommendations are personal, positive and actionable.

- is educational - It increases awareness and teaches new, good habits

\section{A New System Architecture and Interaction Model}

The new and simplified system architecture is described in Fig. 8. In this architecture, we simplified the original one (see Fig. 1) based on the consideration described in above A and B. So that, there is only one application to make UI more simple, however the database is almost same since personal data and logged data such as food and exercise is almost same. In addition, we designed new system using email more actively, not only to provide health care message but also encourage user to input data such as food and exercise. The detail of implementation was almost the same as the previous version described in Tab. II except Flash Lite was not used in this version to realize simplicity.

A thorough and engaging setup experience, to lay the foundation for a personalized health plan, including diet, nutrition and exercise

- Categorized, simplified data-entry model:

- Time of day: Morning, Afternoon, Evening

- Type of entry: Meal, Drink, Snack, Activity 


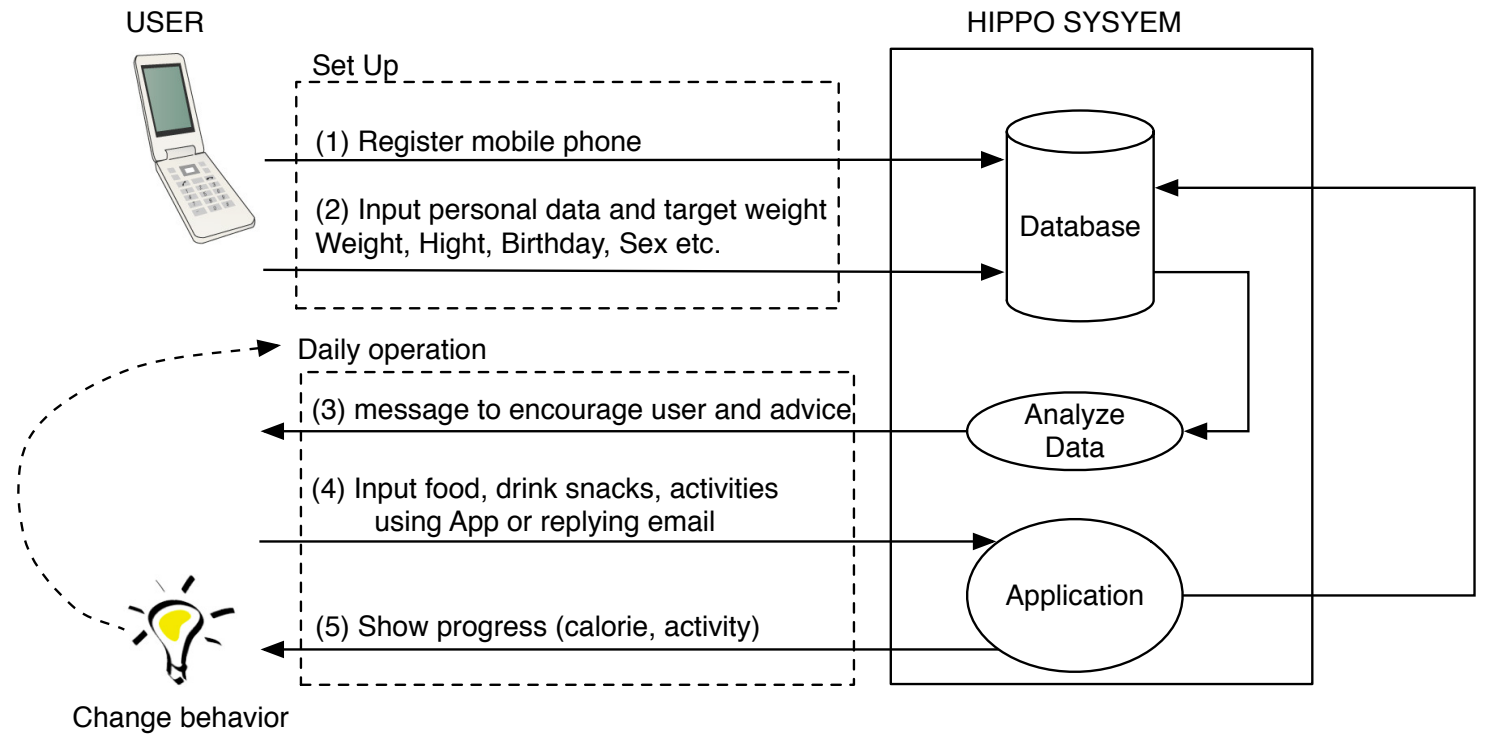

Fig. 8. A new system architecture

- Amount: None, Light, Medium, Heavy, Custom (enter calories), Detailed

- More than 1 type of interaction on a page, making the experience feel quick and responsive

- An interaction model for the User Experience of HIPPO on the web, based on the phone experience, was designed to enhance the experience of setup and data entry.

A new visual style and icon language

- Rich background images and layered UI to create a sense of depth

- Abstract, colorful icons for navigation and data entry

Recommendations for future design and implementation

- List of ideas and opportunities for HIPPO, short- and long-term

The basic interaction model for data input is described in Fig. 9.

Regarding quick data entry, the goal is to capture data quickly and in an engaging fashion

- Data entry is divided up between morning, afternoon and evening, to make it easier to remember food, and better distribute food and exercise throughout the day.

- Entry is categorized into meals, drinks, snacks and exercise, to avoid drinks and snacks being overlooked, and recommend a better, balanced diet plan or menu. Entry is dish-based, instead of food part/component based.

- The system is flexible: alcohol can be entered as afternoon or evening drinks. After-work-snacks can be entered as afternoon or evening snacks, or meals. It is up to the user.

- To educate the user about what light, medium or heavy means, HIPPO shows examples. This example is different every time the user views or selects an amount. The example may include a single food or drink item, or include quantity. For example 2 glasses of orange juice.
For activities "intensity" is presented. Examples come in the form of distance or duration. For example: a 20 minute walk, or a $5 \mathrm{~km}$ run.

As described in sample screens in Fig. 9A, weight is displayed on this screen instead of on the HIPPO home page. This is in line with the current hierarchy of steps, and preserves peoples' privacy. When the selection changes, the focus moves to the next item in the list.

Although this looks like 2 screens, this is a single screen with the focus changing from the top section to the bottom section. The top section contains an instruction and 4 items in carousel (Meal, Drink, Snack, Exercise). By using the left and right buttons on the phone, the user can cycle through the options in the carousel. When the user decides to enter Meal and has the meal icon in the center, he/she clicks OK. This confirms the entry, reflected in the text (Selected: Meal) and the focus is now on selecting and amount (Fig. 9B).

About Detailed entry (Fig. 9C), make reasonable assumptions to reduce the number of (irrelevant) choices

- we know that the user does not eat meat, HIPPO should hide meat-based dishes

- we know that the user is entering Morning meals, HIPPO should de-prioritize meals and dishes that are typical for lunches and dinners.

- Let users enter information on a dish basis: instead of entering bread, butter and cheese separately, provide Sandwich as a choice. By default HIPPO can show a bacon lettuce and tomato sandwich, and let the user adjust the toppings of the sandwich or the type of bread.

About Reminders and Emails, make reminders actionable, personal and positive

- Positive messages especially when things do not go so well (for example the user has gained weight), an encouraging message will likely have more effect.

- Women might prefer different types of encouragement than men 


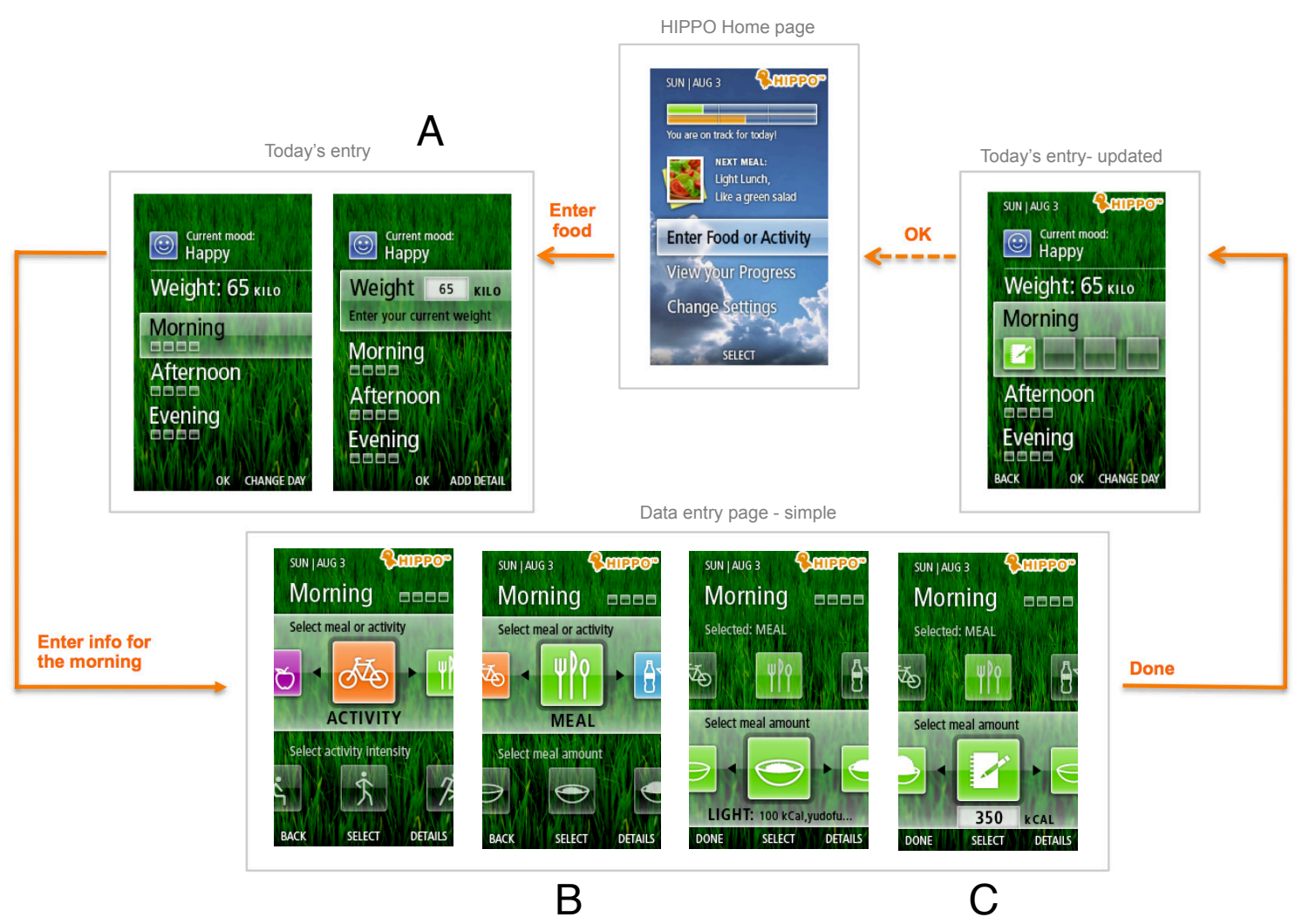

Fig. 9. Basic interaction model for data input

- Variety - prevent that reminders and messages becoming too predictive. A light-hearted, unexpected message can come as a pleasant surprise (a repetitive message may quickly be perceived as nagging) and will ensure that the experience lasts...

- Find out (or learning from the input data) what the difficult moments in the day are, or which foods the user likes but should not eat too much of. Use this to determine when to send the email reminder, and what the message contains: an alternative food, a quick pep-talk etc.

- Do not encourage reward eating (if you skip breakfast and lunch, you can eat all you want for dinner; if you skip dinner, you can drink 5 glasses of alcohol) - this goes against establishing healthy, sustainable eating patterns.

- Focus on HEALTH (and improvement in health through HIPPO) instead of weight. Health is a bigger, more common goal that many people can relate to: all people want to feel healthy. Weight is only an aspect of a user's health, and it is often not something a person is proud of.

- People that are made to lose weight are less likely to succeed than people who WANT to lose weight. Finding out what motivates people or what they themselves see as the advantages of losing weight (live longer, look better, wear certain clothes, save money, better physical condition, increased self confidence, better love life, eat healthier/tastier food) will allow HIPPO to show personalized messages, reminders and graphs.
The second generation application was also designed as a Web service to be used on every mobile phones.

\section{Trial of the Second Generation Application}

The trial using the second generation application was performed from December 19, 2008 to March 31, 2009.

1) The number of users: The number of applicants was 314, this is $2.7 \%$ of employees. Also, the number of registered users at the end of March was 281.

2) Location/Situation to use this system: We asked users where and when they used HIPPO. One-hundred-twenty-eight users answered this question. at Home, at Night is most popular and Free time and in the train are also popular. In the Fig. 10, the difference between the first trial and the second trial. We assume that the number of users who record weight at night was increased in the second trial.

3) Requests from users: Easier operation is required as described in Fig. 11. Other requests are as follows.

- Automatic input of Calories

- Customizing input data

- Much fun (such as RPG)

- Receive advice from HIPPO

4) The number of days to continue: Figure 12 compares the difference in the trend of users who gave up using HIPPO. In 2007 , very few users remained at the end of the trial, however, in 2008, the number of drop outs at the initial stage was almost the same, but the dropout rate after the second week was lower 


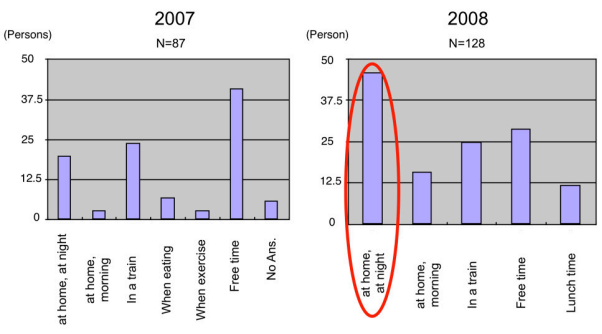

Fig. 10. Location/Situation to use this system.

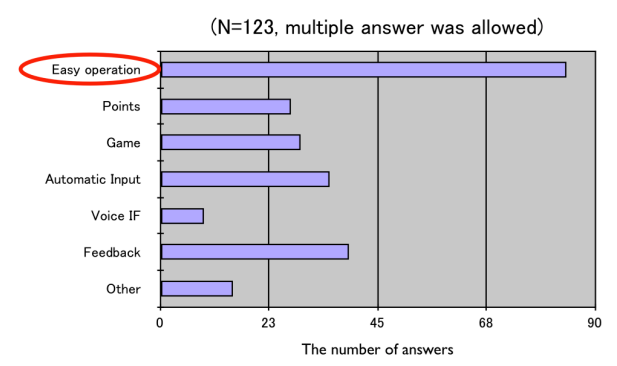

Fig. 11. Requests from users.

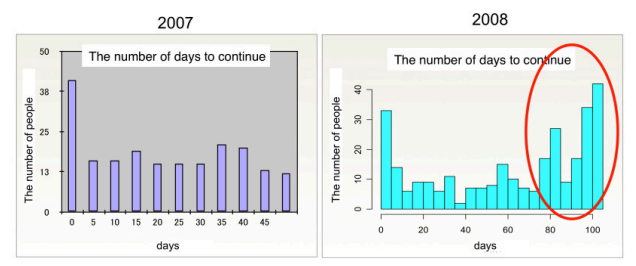

Fig. 12. The number of days to continue.

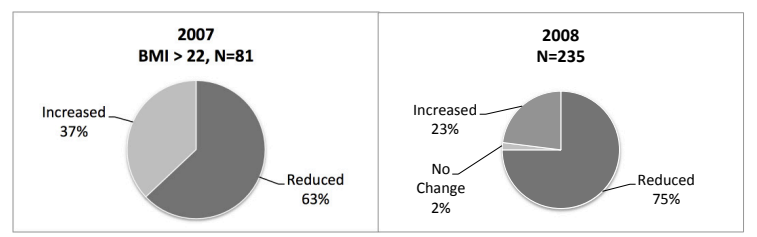

Fig. 13. Weight change.

than that in the trial in 2007. We think that this is the result of the simplified UI of HIPPO.

5) Weight change: Of the 281 registered users, 235 measured their weight. Seventy-eight percent of users who measured their weight lost weight (Fig. 13). This result is better than that of the trial in 2007 . We assume that one of the reasons for the higher number of users who lost weight was the easier operation of the UI.

6) Favorite function: We asked users what is their favorite function. The answers are shown in Fig. 14. Measure weight and record is the most favorite function. Also the answer shows that usability of other functions were also increased. In this questionaire, we received a request for personalized exercise items, automatic calculation of incoming calories.

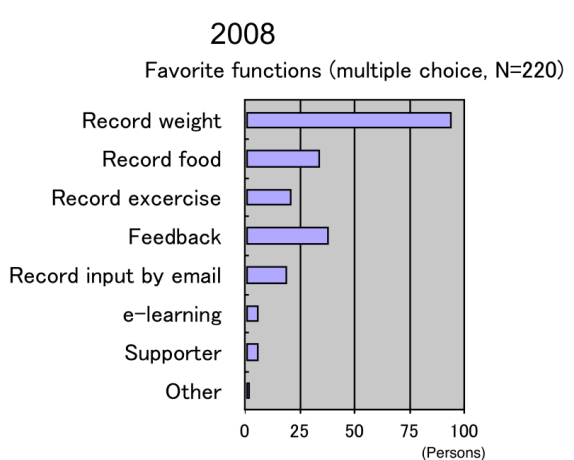

Fig. 14. Favorite function.

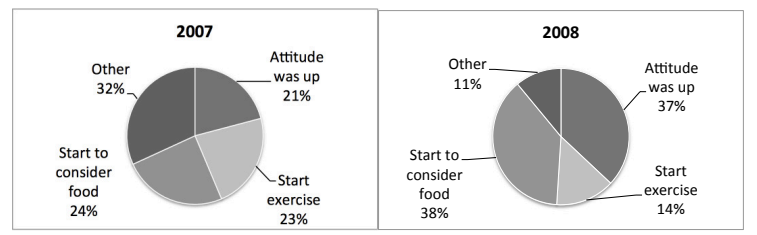

Fig. 15. Change of attitude.

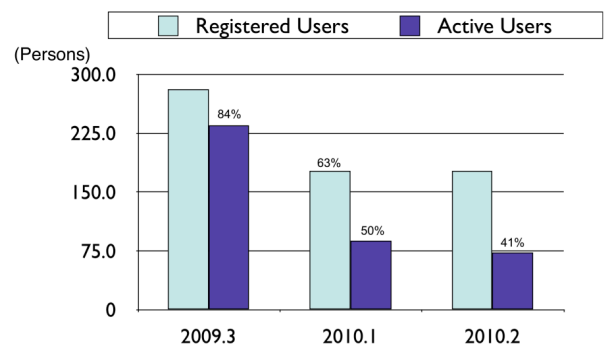

Fig. 16. Retention rate.

7) Change of attitude: Over 90\% of users answered that their attitude was changed as described in Fig. 15. This result was better than that in 2007 .

8) Retention rate: After finishing the second trial, we opened the server for one year. The number of the remaining users is shown in Fig. 16. About $84 \%$ of users used were still using this application at the end of trial period. In another survey, the average retention rate of application in the category of "fitness and health" was about 30\% after 90 days [14], [15]. The retention rate of HIPPO was very high. About 75 users, about $26 \%$ of initial registered users continued to use this application after one year. We assume that this is a very high retention rate.

\section{FUTURE WORKS}

In future, we would like to introduce new features as follows to encourage continued use of the healthcare application.

(1) Automated input and sensors

- Automation of input of health condition into phone: Touch screen phone with vital sensors 
- The scale automatically sends the weight to the phone

- Body suits with vital sensors

- Like Nike+, my shoes, my phone, my heart rate meter, my exercise device in the gym, or my watch send information about my workouts to HIPPO

- Watch-like controller

- Connected on Cloud

- Many sensors installed in house

(2) Online services and events

- The HIPPO challenge: online challenge for individuals or teams to compete in losing weight

- Facebook apps or other groups of support and exchange of experience. You could even "design programs" that you can install and allow people to make food items that are not in the base system.

- Strength workout resource center with short videos and/or info graphics to help users learn proper forms and technique. One could also use the resource center to build and track a comprehensive workout plan. Allowing a personal trainer access to this would also be useful.

- Geography of healthy eating. Especially big cities such as Tokyo, it would be useful to see a visual map of restaurants and food vendors by health, and number of nutritious/low calorie menu items

(3) Community

- Put results in a global perspective: your city, your country, the world

- Put results and accomplishments of the individual in perspective of all users of KDDI : together we have lost $3000 \mathrm{~kg}$, that is $1 \mathrm{SUV}, 500$ dogs, 1000 hamburgers etc.

- Be able to network and partner up with friends or family to create relationships and accountability partners so users do not feel alone. Teams help motivate and push each other to be better.

(4) Alternatives and variety

- Provide alternatives to frequently consumed food to the user, and encourage the user to explore a wider variety of foods

- Users can send recipes/tips/tricks that worked for them to other users? (Sharing information)

- Provide exercise routines or activity suggestions in order to suggest more than just a diet change (really encourage a lifestyle change)- Like you can work out with your phone

- Appeal to younger women or moms and let them use the camera on the phone to research food and nutrition before they buy products. Then find recipes or tips and tricks from other users, with the selected product.

(5) Incentives

- To encourage people to continue with the application, perhaps like a game, there are levels and incentives with unlocking features or gaining new things (maybe you can gain more themes, more characters...). The application evolves and changes as you change your habits with the program. Once you reach a goal, there needs to be additional incentives to encourage people to keep up with the changes they have made.
- Trivia/quiz type game that gets people familiar with how heavy or light certain foods are in calories: it shows you a specific food or drink item and you have to match it to the right calorie count or something along those lines.

\section{CONCLUSION}

In this paper, we compared two applications to support to loose weight and evaluated result of trials, one performed using the first generation and another used the second generation. At first, we developed the first version of mobile application, named HIPPO (Healthcare Information Platform Project), to support to loose weight in 2007. This application worked well during two months and $76 \%$ of users whose BMI was 25 or over lost their weight. However, this problem was that many users could not continue to use this application and users who could continue to use this application could loose weight. We thought that to develop application that makes user continue to use was important. So that, we re-design the architecture and application from three view points, (1) Address real user needs, (2) Rethink and streamline the data-entry model for food and exercise, (3) Make the user experience personal and engaging. We designed one simple application with limited functions and use email as a support tool. Especially, reduction is most important [16] and we proved its effect. As the result, $84 \%$ of uses could continue to use the second generation application.

We learned and proved that reducing the complexity and maintaining motivation were the most important factors for the mobile healthcare application.

\section{ACKNOWLEDGMENT}

We are grateful to the trial participants.

\section{REFERENCES}

[1] "Information of Prevent metabolic syndrome," http://www.mhlw.go.jp/bunya/kenkou/metabo02/other/siryou/index.html.

[2] "Google Health," http://en.wikipedia.org/wiki/Google_health.

[3] "WellnessLINK," http://www.wellnesslink.jp/ (in Japanese).

[4] “KARADAKARUTE," http://www.karadakarute.jp/tanita/ (in Japanese).

[5] “CREATIVE HEALTH," http://www.sankenjin.ne.jp/ (in Japanese).

[6] "Continua Health Alliance," https://quantifiedself.appspot.com/main.jsp.

[7] "Paco," https://quantifiedself.appspot.com/main.jsp.

[8] B. J. Fogg, Persuasive Technology. Morgan Kaufmann, 2002.

[9] Y. de Kort, W. I. Jsselsteijn, C. Midden, B. Eggen, and B. J. Fogg, Persuasive Technology (Second International Conference on Persuasive Technology, PERSUASIVE 2007), 3rd ed. Springer, 2007.

[10] B. J. Fogg and D. Eckles, Mobile Persuasion. Stanford Captology Media, Stanford University, 2007.

[11] B. J. Fogg and R. Adler, Texting 4 Health. Stanford Captology Media, 2009.

[12] https://www.brewmp.com/.

[13] http://www.adobe.com/support/documentation/en/flashlite/.

[14] H. Sudo, "Determinants of exercise adherence of middle-aged women in the gym," Sports Science Research, vol. 5, pp. 96-107, 2008, (in Japanese).

[15] R. K. Dishman, Exercise Adherence: Its Impact on Public Health. Human Kinetics. SChampaign, IL, 1988.

[16] J. Maeda, The Laws of Simplicity. MIT Press, 2006. 